\title{
Ocular Surface Disease Index in Glaucomatous Patients Treated with Bimatoprost
}

\author{
Italo Giuffrè \\ Department of Ophthalmology, Medical School, Catholic University of Rome, Roma, Italy \\ Email: italogiuffre@libero.it
}

Received 1 January 2014; revised 28 March 2014; accepted 23 April 2014

Copyright (C) 2014 by author and Scientific Research Publishing Inc.

This work is licensed under the Creative Commons Attribution International License (CC BY). http://creativecommons.org/licenses/by/4.0/

(c) (i) Open Access

\begin{abstract}
Objectives: To compare ocular surface changes induced via glaucoma treatment in patients using fixed combinations of bimatoprost $0.03 \%$ /timolol $0.50 \%$, timolol $0.50 \%$ or bimatoprost $0.01 \%$ eye drops. Methods: This is a prospective, one center, open-label clinical trial. It was performed on 60 glaucoma patients between 01-01-2012 and 12-31-2012. These patients were randomly divided in three subgroups: bimatoprost $0.03 \%$ /timolol $0.50 \%$ fixed combination, timolol $0.50 \%$ and bimatoprost $0.01 \%$. The Ocular Surface Disease Index (O.S.D.I.) was evaluated in all the glaucomatous patients of the three subgroups at basal time and after 6 and 12 months. All the results were statistically evaluated by Student t-test and one-way ANOVA. The results were considered statistically significant if $p<0.05$. Results: All of the patients ended the clinical trial. There was no statistical significant difference between patients treated with the bimatoprost $0.03 \% /$ timolol $0.50 \%$ fixed combination and timolol $0.50 \%$ eye drops alone $(p=0.845)$. Instead, there was a statistically significant difference between bimatoprost $0.01 \%$ and bimatoprost $0.03 \% /$ timolol $0.50 \%$ patients $(p=0.05)$ and between bimatoprost $0.01 \%$ and timolol $0.50 \%$ eye drops alone $(p=0.049)$. Conclusions: This is a clinical trial based not on the hypotonising effect of these drugs but on their tolerability. The drug which showed the best tolerability is bimatoprost $0.01 \%$.
\end{abstract}

\section{Keywords}

Bimatoprost 0.01\%, Bimatoprost 0.03\%/Timolol 0.50\% Fixed Combination, Glaucoma, OSDI, Timolol 0.50\%

\section{Introduction}

Glaucoma is a chronic, multifactorial, progressive optic neuropathy that needs long-term treatment with topical 
hypotensive medications. Several classes of drugs are currently available to treat this condition, including notselective and selective beta-blockers, carbonic anhydrase inhibitor, prostaglandin (PG) and prostamide analogues as well as fixed combinations (Fcs) of prostaglandin/prostamide analogues combined with $0.50 \%$ timolol maleate [1]-[3]. In the Literature, there is also a bimatoprost formulated in DuraSite [4]. Bimatoprost 0.01\% and bimatoprost $0.03 \%$ were also compared as adherence and persistence [5] and from an economic point of view [6] [7].

Beta-blockers are often used to treat glaucoma and were considered the "gold standard" for starting glaucoma treatment until recently, when they were replaced by prostaglandin and prostamide analogues. Beta-blockers have many systemic side-effects, including bradycardia, and bronchospasms, as well as effects on the central nervous system [2] [3].

While the systemic side-effects induced by topical PG analogues are rare, iris hyperpigmentation, excessive eyelash growth and conjunctival hyperemia have been reported among the local side-effects caused by these drugs [8]-[10].

Ocular surface dysfunction has also been related to glaucoma treatment. Beta-blockers have been known to induce conjunctival hyperemia, punctate keratitis and corneal anesthesia, as well as dry eye and allergic blepharoconjunctivitis [11]-[13]. Indeed, the lengh of administration, concentration and amount of these drugs have been related to the severity of the side-effects. The purpose of this study is to evaluate the OSDI in glaucomatous patients using bimatoprost $0.03 \%$ /timolol 0.50\% fixed combination (BTFC; Ganfort ${ }^{\circledR}$, Allergan, Irvine, CA, USA), timolol $0.50 \%$ or bimatoprost $0.01 \%$ (Lumigan $0.1^{\circledR}$, Allergan, Irvine, CA, USA) eye drops alone.

\section{Patients and Methods}

\subsection{Inclusion Criteria}

Eligible patients were adults (>18 years of age) with a clinical diagnosis of open-angle glaucoma (OAG) in both eyes and with no previous topical hypotensive treatment. These patients had an open iridocorneal angle upon gonioscopy examination. OAG was diagnosed on the basis of typical optic disc changes and glaucomatous visual field loss demonstrated on the Humphrey visual field analyzer (HFA) (Humphrey Instruments, Inc., Zeiss Humphrey, San Leandro, California, USA). Intraocular pressure (IOP), measured at 8 am, corrected by pachymetry, was over $25 \mathrm{mmHg}$ at baseline. In addition, patients were required to have a corrected distance visual acuity (CDVA) of 20/70 or better in each eye. These patients had a recent (within three months) visual field examination showing a mean deviation greater than-15 dB. Finally, the eligible patients were required to be able to follow instructions, to be willing and able to attend all of the study visits, and to provide informed consent prior to screening.

\subsection{Exclusion Criteria}

Patients were excluded if they met any of the following criteria: previous ocular surgery; active ocular inflammation; or clinically diagnosed dry eye. Patients with hypersensitivity or poor tolerance to any components of the study medication; with bronchial asthma or history of bronchial asthma; with bronchial hyperreactivity or severe chronic obstructive pulmonary disease that would preclude the safe administration of a topical betablocker; sinus bradycardia, second-degree or third-degree atrioventricular block, sinoatrial block, cardiac failure, or cardiogenic shock that would preclude the safe administration of a topical beta-blocker; or a severe medical or psychiatric disease were also excluded from the study.

This was a prospective, single center, open-labeled clinical trial. It was conducted between 01-01-2012 and 12-31-2012 at the Glaucoma Service of the Ophthalmological Department of Catholic University of Roma, Roma, ITALY, EU. Sixty patients (30 M, 30 F) were allocated using permuted-block randomization (block size $=3$; allocation rate $1: 1: 1$ ) into the following three groups: bimatoprost $0.03 \%+$ timolol $0.50 \%$ fixed combination (BTFC) (Group A); timolol 0.50\% (T) (Group B); or bimatoprost 0.01\% (B) (Group C).

The clinical data collected included the patients' demographic data (age and sex). In Group A, there were 10 males and 10 females and the mean age was 64.33, SD 12.03 years (Table 1 ). In Group B the sex rate was also 1:1, 10 males to 10 females and the mean age was 62.67, SD 7.367 years (Table 2). In Group C, there was the same number of males and females (10 M, $10 \mathrm{~F}$ ) and the mean age was 76.21, SD 8.251 years (Table 3). All of the patients underwent routine ophthalmological assessment prior to and after 6 and 12 months of treatment. The 
OSDI questionnaire was applied at each check time.

\subsection{Statistical Analysis}

Student t-test was used to compare the age and pachymetrycal data of these groups. One-way ANOVA with a significance level of $5 \%$ was used to compare the continouous variables. The level of statistical significance was set at $\mathrm{p}=0.05$.

\section{Results}

The results of the statistical analysis were unaffected by the demographic data $(p=0.079)$. All the patients included in this clinical trial ended it and nobody needed to change the hypotonising therapy. The OSDI scores were not statistically significant difference between Group A $(B T F C)$ and B $(T)$ patients $(p=0.845)$ (Table 4). Instead, the OSDI scores were statistically significant different between Group $C$ (B) and Group A (BTFC) patients ( $p=0.05)$ and between Group C (B) and Group B (T) patients ( $=0.049)$ (Table 4) at time 12 months.

\section{Discussion and Conclusions}

Strong evidence provided by previous clinical and experimental studies has indicated that the chronic use of antiglaucoma drugs might induce ocular surface changes, causing discomfort at instillation, conjunctival inflammation, tear film instability, subconjunctival fibrosis, apoptosis of conjunctival epithelial cells and corneal surface changes. These changes could result in a greater risk of failure when patients undergo antiglaucoma surgery, particularly trabeculectomy, as a result of postoperative fibrosis.

Several previous studies prove that changes in cell-to-cell contact result in a loss of "gap junctions" and in edema, which are reflected in the epithelial architecture and which lead to keratopathy. These pathological changes in the ocular surface could partly explain the occurrence of dry eye symptoms in patients using hypotensive drugs.

Some Authors reported that prolonged use (longer than six months) of $0.50 \%$ timolol maleate might lead to a

Table 1. Demographics of Group A.

\begin{tabular}{cc}
\hline PATIENTS & AGE \\
\hline $10 \mathrm{M} ; 10 \mathrm{~F}$ & $64.33 \mathrm{y} ; 12.03$ y SD \\
\hline
\end{tabular}

Table 2. Demographics of Group B.

\begin{tabular}{cc}
\hline PATIENTS & AGE \\
\hline $10 \mathrm{M} ; 10 \mathrm{~F}$ & $62.67 \mathrm{y} ; 7.367 \mathrm{y} \mathrm{SD}$ \\
\hline \multicolumn{2}{|c}{ Table 3. Demographics of Group C. } \\
\hline PATIENTS & AGE \\
\hline 10 M; 10 F & $76.21 \mathrm{y} ; 8.251$ y SD \\
\hline
\end{tabular}

Legenda: M: males; F: females; SD: standard deviation; y: years.

Table 4. Results.

\begin{tabular}{cc}
\hline BTFC vs. T & $\mathrm{p}=0.845$ \\
B vs. BTFC & $\mathrm{p}=0.05$ \\
B vs. T & $\mathrm{p}=0.049$ \\
\hline
\end{tabular}

Legenda: BTFC: Bimatoprost 0.03\%/Timolol 0.50\% Fixed Combination; T: Timolol 0.50\%; B: Bimatoprost 0.01\%. 
higher incidence of dry eye, with lower break-up time (TBUT) values and Schirmer's test scores. Several reports have also demonstrated that timolol could cause a decrease in the number of goblet cells and keratoconjunctivitis sicca. Reduction of TBUT values and Schirmer's test scores have been described in many studies investigating the effects of the length of glaucoma treatment, the number of medications used and preservative concentrations.

More recently, some authors, as we did, have used the OSDI (Ocular Surface Disease Index) questionnaire, a useful and economic tool, downloaded by web, for analyzing symptoms of dry eye, to measure symptoms in glaucoma patients, not considering the visual field index [14]. The lower the OSDI score is, the less toxic the medication is.

In summary, this study demonstrated that bimatoprost $0.01 \%$ is quite less toxic to the ocular surface after 12 months exposure than bimatoprost $0.03 \% /$ timolol $0.50 \%$ fixed combination and timolol $0.50 \%$ eye drops alone.

\section{Footnotes}

The author declares to have no conflict of interest in any of the drug mentioned in this study.

\section{References}

[1] Russ, H.H., Nogueira-Filho, P.A., Barros Jde, N., et al. (2013) Ocular Surface Evaluation in Patients Treated with a Fixed Combination of Prostaglandin Analogues with 0.5\% Timolol Maleate Topical Monotherapy: A Randomized Clinical Trial. Clinics (Sao Paulo), 68, 1318-1324. http://dx.doi.org/10.6061/clinics/2013(10)05

[2] Nixon, D.R. (2013) A Randomized, Prospective Study of Bimatoprost 0.01\% or Travoprost/Timolol in Patients Previously Treated with Latanoprost and Timolol to Reduce Intraocular Pressure. Journal of Ocular Pharmacology and Therapeutics, 29, 876-881. http://dx.doi.org/10.1089/jop.2013.0108

[3] Pfennigsdorf, S., de Jong, L., Makk, S., et al. (2013) A Combined Analysis of Five Observational Studies Evaluating the Efficacy and Tolerability of Bimatoprost/Timolol Fixed Combination in Patients with Primary Open-Angle Glaucoma or Ocular Hypertension. Journal of Clinical Ophthalmology, 7, 1219-1225.

http://dx.doi.org/10.2147/OPTH.S41885

[4] Shafiee, A., Bowman, L.M., Hou, E., et al. (2013) Ocular Pharmacokinetics of Bimatoprost Formulated in DuraSite Compared to Bimatoprost 0.03\% Ophthalmic Solution in Pigmented Rabbit Eyes. Journal of Clinical Ophthalmology, 7, 1549-1556. http://dx.doi.org/10.2147/OPTH.S48766

[5] Campbell, J.H., Schwartz, G., Labounty, B., et al. (2013) Comparison of Adherence and Persistence with Bimatoprost 0.01\% versus Bimatoprost 0.03\% Topical Ophthalmic Solutions. Current Medical Research and Opinion, 29, 12011209. http://dx.doi.org/10.1185/03007995.2013.815160

[6] Wong, W.B., Patel, V.D., Kowalski, J.W., et al. (2013) An Adherence Based Cost-Consequence Model Comparing Bimatoprost 0.01\% to Bimatoprost 0.03\%. Current Medical Research and Opinion, 29, 1191-1200. http://dx.doi.org/10.1185/03007995.2013.815159

[7] Alany, R.G. (2013) Adherence, Persistence and Cost-Consequence Comparison of Bimatoprost Topical Ocular Formulations. Current Medical Research and Opinion, 29, 1187-1189. http://dx.doi.org/10.1185/03007995.2013.818968

[8] Stewart, W.C., Kolker, A.E., Stewart, J.A., et al. (2003) Conjunctival Hyperemia in Healthy Subjects after Short-Term Dosing with Latanoprost, Bimatoprost, and Travoprost. American Journal of Ophthalmology, 135, 314-320. http://dx.doi.org/10.1016/S0002-9394(02)01980-3

[9] Johnstone, M.A. (1997) Hypertrichosis and Increased Pigmentation of Eyelashes and Adjacent Hair in the Region of the Ipsilateral Eyelids of Patients Treated with Unilateral Topical Latanoprost. American Journal of Ophthalmology, 124, 544-547.

[10] Kook, M.S. and Lee, K. (2000) Increased Eyelid Pigmentation Associated with the Use of Latanoprost. American Journal of Ophthalmology, 129, 804-806. http://dx.doi.org/10.1016/S0002-9394(00)00402-5

[11] Herreras, J.M., Pastor, J.C., Calonge, M., et al. (1992) Ocular Surface Alteration after Long-Term Treatment with Antiglaucomatous Drug. Ophthalmology, 99, 1082-1088. http://dx.doi.org/10.1016/S0161-6420(92)31847-0

[12] Nuzzi, R., Finazzo, C. and Cerruti, A. (1998) Adverse Effects of Topical Antiglaucomatous Medications on the Conjunctiva and the Lacrymal. International Ophthalmology, 22, 31-35. http://dx.doi.org/10.1023/A:1006051725115

[13] Broadway, D.C., Grierson, I., O’Brien, C., et al. (1994) Adverse Effects of Topical Antiglaucoma Medication. The Conjunctival Cell Profile. Archives of Ophthalmology, 112, 1437-1445. http://dx.doi.org/10.1001/archopht.1994.01090230051020

[14] Arici, M.K., Arici, D.S., Topalkara, A., et al. (2000) Adverse Effects of Topical Antiglaucoma Drugs on the Ocular Surface. Journal of Clinical \& Experimental Ophthalmology, 28, 113-117. http://dx.doi.org/10.1046/j.1442-9071.2000.00237.x 